\title{
Distributed Event-Based Sliding-Mode Consensus Control in Dynamic Formation for VTOL-UAVs
}

\author{
J. U. Alvarez-Muñoz ${ }^{1}$, J. Chevalier ${ }^{1}$, Jose J. Castillo-Zamora ${ }^{2}$ and J. Escareno ${ }^{3}$
}

\begin{abstract}
The present work deals with consensus control for a multi-agent system composed by mini Vertical Takeoff and Landing (VTOL) rotorcrafts by means of a novel nonlinear event-based control law. First, the VTOL system modeling is presented using the quaternion parametrization to develop an integral sliding-mode control law for attitude stabilization of the aerial robots. Then, the vehicle position dynamics is expanded to the multi-agent case where a cuttingedge event-triggered sliding-mode control is synthesized to fulfill the collective consensus objective within a formation context. With its inherent robustness and reduced computational cost, the aforementioned control strategy guarantees closed-loop stability, while driving trajectories to the equilibrium in the presence of time-varying disturbances. Finally, for validation and assessment purposes of the overall consensus strategy, an extensive numerical simulation stage is conducted.
\end{abstract}

\section{INTRODUCTION}

In recent years, the technological surge in terms of MultiAgent Systems (MAS) and the control theory behind made possible the usage of these systems for multiple applications in different sectors, including transportation, manipulation and rescue operations. Since then, swarm control of autonomous systems features various functions, such as the consensus, where an agreement between the agents to recognize the states of each individual of the system is required [1], [2]. In addition to consensus, the formation control allows to ensure large-scale multi-agent systems dynamic viability [3]. But in some cases, due to communication or processing limitations, distributed models are preferable [4]-[6]. The distributed formation control approach has been verified under various conditions, such as the formation of shapes in the 3 dimensional space [7] or interconnection graph changes [8].

However, large-scale swarms of autonomous systems face the problem of limited communication bandwidth. Even more, when several tasks such as remote control or video transmitting work at the same time, deterioration of controllers performance can arise. To solve this issue, the event-based paradigm emerged. The idea is to compute and update the control signals only when an event occurs. Mathematical models and simulation of single and double integrator agents show the relevance of event-based controllers regarding communication delays, packet drops or noise [9][11]. Applied to similar systems, this approach proves also

*Corresponding author: jalvarezmunoz@extia.fr

${ }^{1}$ J. U. Alvarez-Muñoz and J. Chevalier are with EXTIA, Sevres, 92310, France

${ }^{2}$ Jose J. Castillo-Zamora is with Université Paris Saclay, Laboratory of Signals and Systems, CNRS-CentraleSupelec, Gif-sur-Yvette, and IPSA Paris, Ivry-sur-Seine, France

${ }^{3} \mathrm{~J}$. Escareno is with UENSIL-ENSCI, Limoges, CNRS, XLIM, UMR 7252, Limoges, France its effectiveness during obstacle avoidance while maintaining formation as the simulated results shown in [12]. In addition, experimental validation of a group of mini VTOL-UAVs confirmed the performances of such control systems regarding the communication bandwidth preservation [13].

These works ensure consensus and formation control with bandwith usage reduction. However, for applications where unknown disturbances may be present, robust controllers need to be implemented. In this context, the Sliding-Mode Control (SMC) [14], [15], which is known for its inherent robustness can be implemented in multi-agent systems. On this subject, an adaptive sliding mode control law was developed and proved through simulation results [16], where the flight stability of a group of VTOL-UAVs exposed to constant and bounded disturbances was improved. The results validate the relevance of such a control law even when applied to the non-linear dynamics of VTOL-UAVs. Therefore, it is interesting to combine the two features presented before to increase the performance of aerial swarm systems.

As an example of that, [17] deals with the leaderfollowing consensus problem from an event-based sliding mode controller perspective. The design of the SMC for time-finite consensus is addressed and extended to an eventbased implementation. A nonlinear second-order multi-agent system is presented in [18] where an integral sliding mode surface and an event-mechanism for the controller update are formulated. Both works validate their proposals through formal mathematical analyisis and the results of nonlinear double-integrator multi-agent systems.

Captivated by the aforementioned works, the actual paper presents a proposal regarding the coordinating control of a set of mini VTOL rotorcrafts by designing an event-based and adaptive SMC. For this, the inner-outer loop control methodology is implemented. First, and contrary to most of the approaches cited above which use Euler angles, a robust control technique consisting of an Integral Sliding-Mode Control (ISMC) based on the quaternion parametrization for each VTOL rotorcraft is designed to ensure attitude stabilization. Then, the present work explains the construction of a robust collaborative position control scheme, composed of a sliding-mode surface, an adaptive term and a triggermechanism regarding the outer loop control. The main idea behind this approach is to take advantage of the features present in the research previously cited into one control algorithm. Practical convergence to the leader in terms of position and velocity, robustness to bounded disturbances, reduction in terms of energy consumption and inter-vehicles communication are demonstrated through this work. The 
effectiveness of the proposal is demonstrated through a formal stability analysis and a detailed simulation scenario with five mini VTOL-UAVs, subjected to continuous and time-varying disturbances.

The sequel of the paper is structured as follows. In Section II, some mathematical preliminaries used throughout the manuscript are presented. Section III is devoted to the mathematical modeling of the VTOL-UAV system. Section IV presents the attitude control law for each robot, the formulation of the event-triggered control law and the consensus strategy for the set of aerial vehicles. The simulation scenario and numerical results are presented in Section V. The conclusions and future work are presented in Section VI.

\section{TheoreticAl PREREQUisites}

The current section presents the mathematical concepts of graph theory, quaternion representation and event-triggered control used throughout the paper.

\section{A. Graph Theory}

A MAS can be modeled as a set of dynamic systems (or agents) in which an information exchange occurs. Such information flow is mathematically represented by means of graph theory. In this regard, let $\mathcal{G}=\{\mathcal{V}, \xi\}$ be defined by the sets $\mathcal{V}=1, \ldots, N$ and $\xi$ which represents the vertices (or nodes) and edges of the graph, respectively. Adjacency between two nodes, $i$ and $j$, exists if there is an edge $(i, j)$ that connects both nodes. In this sense, such nodes are said to be adjacent and the aforementioned relation is formally represented as:

$$
\xi=(i, j) \in \mathcal{V} \times \mathcal{V}: i, j
$$

An undirected graph is described as a graph where the node $i$ can obtain information about the node $j$ and vice versa, i.e.

$$
(i, j) \in \xi \Leftrightarrow(j, i) \in \xi
$$

The matrix $\mathcal{A}$ is called the adjacency matrix and its elements $a_{i j}$ describe the adjacency between nodes $i$ and $j$ such that

$$
a_{i j}=\left\{\begin{array}{cc}
1 & i \text { and } j \text { are adjacent } \\
0 & \text { otherwise }
\end{array}\right.
$$

If all pairs of nodes in $\mathcal{G}$ are connected, then $\mathcal{G}$ is called connected. The distance $d(i, j)$ is defined by the shortest path between nodes $i$ and $j$, and it is equal to the number of edges that conform the path. The degree matrix $\mathcal{D}$ of $\mathcal{G}$ is the diagonal matrix with elements $d_{i}$ equal to the cardinality of node $i$ 's neighbor set $N_{i}=j \in V:(i, j) \in \xi$. The Laplacian matrix $\mathcal{L}$ of $\mathcal{G}$ is defined as $\mathcal{L}=\mathcal{D}-\mathcal{A}$. For undirected graphs, $\mathcal{L}$ is symmetric and positive semi-definite, i.e., $\mathcal{L}=\mathcal{L}^{T} \geq 0$. Moreover, the row sums of $\mathcal{L}$ are zero. For connected graphs, $\mathcal{L}$ has exactly one zero eigenvalue, and the eigenvalues can be listed in increasing order $0=\lambda_{1}(\mathcal{G})<\lambda_{2}(\mathcal{G}) \leq \ldots \leq$ $\lambda_{N}(\mathcal{G})$. The second eigenvalue $\lambda_{2}(\mathcal{G})$ is called the algebraic connectivity.
If the system has a leader-following configuration, the leader is represented by an extra vertex 0 , and then communication between the leader and the followers is performed. $\mathcal{B}$ is then a diagonal matrix representing this communication, with entries 1 , if there exists an edge between the leader and any other agent in the group, or 0 , otherwise.

Lemma 2.1: The matrix $\mathcal{L}+\mathcal{B}$ has full rank when $\mathcal{G}$ has a spanning tree with leader as the root, which implies non singularity of $\mathcal{L}+\mathcal{B}$

Remark 2.2: From here, we shall refer to the matrix $\mathcal{L}+\mathcal{B}$ as $\mathcal{H}$, in order to avoid any confusion.

\section{B. Unit Quaternion and Attitude Kinematics}

Considering two orthogonal right-handed coordinate frames: the body coordinate frame, $B\left(x_{b}, y_{b}, z_{b}\right)$, located at the center of mass of a rigid body and the inertial coordinate frame, $N\left(x_{n}, y_{n}, z_{n}\right)$, located at some point in the space (for instance, the earth NED frame). The rotation of the body frame $B$ with respect to the fixed frame $N$ is represented by the attitude matrix $R \in S O(3)=\left\{R \in \mathbb{R}^{3 \times 3}: R^{T} R=\right.$ $I_{3}$, det $\left.R=1\right\}$.

The cross product between two vectors $\xi, \varrho \in \mathbb{R}^{3}$ is represented by a matrix multiplication $\left[\xi^{\times}\right] \varrho=\xi \times \varrho$, where $\left[\xi^{\times}\right]$is the well-known skew-symmetric matrix. The $n$-dimensional unit sphere embedded in $\mathbb{R}^{n+1}$ is denoted as $\mathbb{S}^{n}=\left\{x \in \mathbb{R}^{n+1}: x^{T} x=1\right\}$. Members of $S O(3)$ are often parameterized in terms of a rotation $\beta \in \mathbb{R}$ about a fixed axis $e_{v} \in \mathbb{S}^{2}$ by the map $\mathcal{U}: \mathbb{R} \times \mathbb{S}^{2} \rightarrow S O(3)$ such that

$$
\mathcal{U}\left(\beta, e_{v}\right):=I_{3}+\sin (\beta)\left[e_{v}^{\times}\right]+(1-\cos (\beta))\left[e_{v}^{\times}\right]^{2}
$$

Hence, a unit quaternion, $q \in \mathbb{S}^{3}$, is defined as

$$
q:=\left(\begin{array}{c}
\cos \frac{\beta}{2} \\
e_{v} \sin \frac{\beta}{2}
\end{array}\right)=\left(\begin{array}{c}
q_{0} \\
q_{v}
\end{array}\right) \in \mathbb{S}^{3}
$$

where $q_{v}=\left(\begin{array}{lll}q_{1} & q_{2} & q_{3}\end{array}\right)^{T} \in \mathbb{R}^{3}$ and $q_{0} \in \mathbb{R}$ are known as the vector and scalar parts of the quaternion respectively. The quaternion $q$ represents an element of $S O(3)$ through the map $R: \mathbb{S}^{3} \rightarrow S O(3)$ defined as

$$
R:=I_{3}+2 q_{0}\left[q_{v}^{\times}\right]+2\left[q_{v}^{\times}\right]^{2}
$$

Remark 2.3: $R=R(q)=R(-q)$ for each $q \in \mathbb{S}^{3}$, i.e. even quaternions $q$ and $-q$ represent the same physical attitude.

Denoting by $\vec{\omega}=\left(\begin{array}{lll}\omega_{1} & \omega_{2} & \omega_{3}\end{array}\right)^{T}$ the angular velocity vector of the body coordinate frame, $B$ relative to the inertial coordinate frame $N$ expressed in $B$, the kinematics equation is given by

$$
\left(\begin{array}{c}
\dot{q}_{0} \\
\dot{q}_{v}
\end{array}\right)=\frac{1}{2}\left(\begin{array}{c}
-q_{v}^{T} \\
I_{3} q_{0}+\left[q_{v}^{\times}\right]
\end{array}\right) \boldsymbol{\omega}=\frac{1}{2} \Xi(q) \boldsymbol{\omega}
$$

The attitude error is used to quantify mismatch between two attitudes. If $q$ defines the current attitude quaternion and $q_{d}$ the desired quaternion, i.e. the desired orientation, then the error quaternion that represents the attitude error between the current orientation and the desired one is given by

$$
q_{e}:=q_{d}^{-1} * q=\left(q_{e_{0}} q_{e_{v}}^{T}\right)^{T}
$$




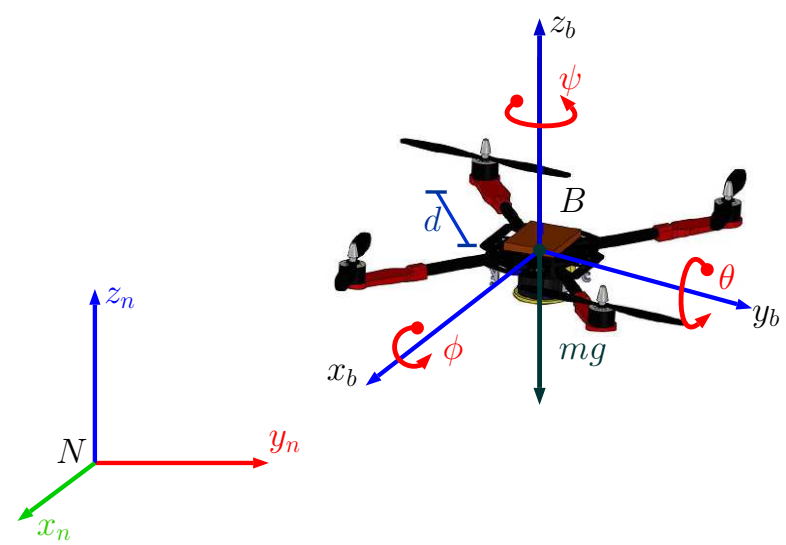

Fig. 1: Schematic configuration of a VTOL vehicle in the $3 D$ space.

where $q^{-1}$ is the complementary rotation of the quaternion $q$ which is given by $q^{-1}:=\left(\begin{array}{ll}q_{0} & -q_{v}^{T}\end{array}\right)^{T}$ and $(*)$ denotes the quaternion multiplication.

\section{Attitude And Position Dynamics of the VTOL Multi-Agent System}

If a group of $N$-VTOL vehicles is considered and each aerial system is modeled as a rigid body, as in Fig. 3, then, according to [19], the six degrees of freedom model (position and orientation) of the system can be separated into translational and rotational motions, defined respectively by

$$
\begin{aligned}
& \Sigma_{T_{i}}:\left\{\begin{array}{l}
\dot{\boldsymbol{p}}_{i}=\boldsymbol{v}_{i} \\
m_{i} \dot{\boldsymbol{v}}_{i}=-m_{i} \boldsymbol{g}+R_{i}\left(\begin{array}{c}
0 \\
0 \\
U_{T_{i}}
\end{array}\right)+\varsigma_{i}
\end{array}\right. \\
& \Sigma_{R_{i}}:\left\{\begin{array}{l}
\dot{q}_{i}=\frac{1}{2} \Xi\left(q_{i}\right) \boldsymbol{\omega}_{\boldsymbol{i}} \\
J_{i} \dot{\boldsymbol{\omega}}_{i}=-\boldsymbol{\omega}_{i}^{\times} J_{i} \boldsymbol{\omega}_{i}+\boldsymbol{\Gamma}_{i}
\end{array}\right.
\end{aligned}
$$

where $i=1, \ldots, N . \boldsymbol{p}_{i}$ and $\boldsymbol{v}_{i}$ are linear positions and velocities vectors, $m_{i}$ is the mass of each aerial system, $\boldsymbol{g}$ is the gravity vector, $R$ is the rotation matrix given in (3), $U_{T_{i}}$ is the total thrust and $\varsigma_{i}$ corresponds to an unknown disturbance, bounded in the manner $\left\|\varsigma_{i}\right\| \leq \varsigma_{\max }$. Besides, $J_{i} \in \mathbb{R}^{3 \times 3}$ is the inertia matrix of the rigid bodies expressed in the body frame $B$ and $\boldsymbol{\Gamma}_{i} \in \mathbb{R}^{3}$ is the vector of applied torques. $\boldsymbol{\Gamma}_{i}$ depends on the (control) couples generated by the actuators and the aerodynamics, such as gyroscopic couples or the gravity gradient.

Note that the rotation matrix $R$ can also be defined according to the Euler angles $\phi, \theta, \psi$, correspondingly referred to as roll, pitch and yaw angles

$$
\begin{aligned}
& R(\phi, \theta, \psi)= \\
& \left(\begin{array}{ccc}
\mathrm{C}_{\theta} \mathrm{C}_{\psi} & \mathrm{S}_{\phi} \mathrm{S}_{\theta} \mathrm{C}_{\psi}-\mathrm{C}_{\phi} \mathrm{S}_{\psi} & \mathrm{C}_{\phi} \mathrm{S}_{\theta} \mathrm{C}_{\psi}+\mathrm{S}_{\phi} \mathrm{S}_{\psi} \\
\mathrm{C}_{\theta} \mathrm{S}_{\psi} & \mathrm{S}_{\phi} \mathrm{S}_{\theta} \mathrm{S}_{\psi}+\mathrm{C}_{\phi} \mathrm{C}_{\psi} & \mathrm{C}_{\phi} \mathrm{S}_{\theta} \mathrm{S}_{\psi}-\mathrm{S}_{\phi} \mathrm{C}_{\psi} \\
-\mathrm{S}_{\theta} & \mathrm{C}_{\theta} \mathrm{S}_{\phi} & \mathrm{C}_{\theta} \mathrm{C}_{\phi}
\end{array}\right)
\end{aligned}
$$

where $S_{\star}$ and $C_{\star}$ stand for $\sin (\star)$ and $\cos (\star)$, respectively.

\section{Attitude And Position Control for the VTOL MAS}

The current section is divided in two parts. First, we introduce the attitude control law to stabilize the $i^{\text {th }}$ agent's attitude, followed by the position control strategy to achieve convergence to the leader and multi-agent formation.

\section{A. Attitude Stabilization Method}

The aim of this section is to present the design procedure of an attitude control which drives the aerial vehicles to attitude stabilization, i.e. to the asymptotic conditions below

$$
q_{i} \rightarrow\left[\begin{array}{llll} 
\pm 1 & 0 & 0 & 0
\end{array}\right]^{T}, \boldsymbol{\omega}_{\boldsymbol{i}} \rightarrow 0 \text { as } t \rightarrow \infty
$$

The angular velocity error for each aerial vehicle in terms of quaternions is given by the next expression

$$
\boldsymbol{\omega}_{e i}=\boldsymbol{\omega}_{i}-R_{i} \boldsymbol{\omega}_{d i}
$$

where $\boldsymbol{\omega}_{i}$ corresponds to the actual orientation of the system and $R_{i}$ is the rotation matrix given by (3). Then, by calculating the time derivative of the error quaternion given in (5) and the angular velocity error, the attitude error dynamics can be given by

$$
\begin{gathered}
\left(\begin{array}{c}
\dot{q}_{e i_{0}} \\
\dot{q}_{e i_{v}}
\end{array}\right)=\frac{1}{2}\left(\begin{array}{c}
-q_{e i_{v}}^{T} \\
I_{3} q_{e i_{0}}+\left[q_{e i_{v}}^{\times}\right.
\end{array}\right) \boldsymbol{\omega}_{e i} \\
\dot{\boldsymbol{\omega}}_{e i}=-J_{i}^{-1} \boldsymbol{\omega}_{e i}^{\times} J_{i} \boldsymbol{\omega}_{e i}+J_{i}^{-1} \boldsymbol{\Gamma}_{i}
\end{gathered}
$$

The design of the attitude control law consists of an integral sliding mode control, where the sliding surface is proposed as follows

$$
s_{i}=J_{i} \boldsymbol{\omega}_{e i}+\lambda_{i} q_{e i_{v}}+K_{i} \varepsilon_{i}
$$

where $s_{i} \in \mathbb{R}^{3}, \varepsilon_{i}$ corresponds to the integral of the error in terms of quaternions and $\lambda_{i}$ and $K_{i}$ are constant positive parameters. The time derivative of the previous equation is given by

$$
\dot{s}_{i}=J_{i} \dot{\boldsymbol{\omega}}_{e i}+\lambda_{i} \dot{q}_{e i_{v}}+K_{i} q_{e i_{v}}
$$

Substituting equation (12) into (14), the next expression is obtained

$\dot{s}_{i}=\lambda_{i} \dot{q}_{e i_{v}}+K q_{e i_{v}}+J_{i}\left(\boldsymbol{\omega}_{e i}^{\times} R_{i} \boldsymbol{\omega}_{d i}-R_{i} \dot{\boldsymbol{\omega}}_{d i}\right)-\boldsymbol{\omega}_{i}^{\times} J_{i} \boldsymbol{\omega}_{i}+\Gamma_{i}$

Then, the control law, using the exponential reaching law $\dot{s}=a \operatorname{sign}(s)+b s$, where $a, b>0$ is given by

$$
\begin{aligned}
\boldsymbol{\Gamma}_{i}= & -\lambda_{i} \dot{q}_{e i_{v}}-K_{i} q_{e i_{v}}-J_{i}\left(\boldsymbol{\omega}_{e i}^{\times} R_{i} \boldsymbol{\omega}_{d i}-R_{i} \dot{\boldsymbol{\omega}}_{d i}\right) \\
& +\boldsymbol{\omega}_{i}^{\times} J_{i} \boldsymbol{\omega}_{i}-a_{i} \operatorname{sign}\left(s_{i}\right)-b_{i} s_{i}
\end{aligned}
$$

Finally, in order to reduce the chattering phenomenon, the sign function is replaced by the hyperbolic tangent function as follows: $\operatorname{sign}(s)=\tanh \left(\frac{s}{\alpha}\right)$, with $\alpha$ a small constant to control the shape of the function.

Proof: Let us consider the next candidate Lyapunov function, which is positive-definite:

$$
V=\frac{1}{2} s_{i}^{T} s_{i}
$$


By finding its time derivative and substituting (15) into this one it is possible to obtain

$$
\begin{aligned}
\dot{V}= & s_{i}^{T}\left(\lambda_{i} \dot{q}_{e i_{v}}+K_{i} q_{e i_{v}}+J_{i}\left(\boldsymbol{\omega}_{e i}^{\times} R_{i} \boldsymbol{\omega}_{d i}-R_{i} \dot{\boldsymbol{\omega}}_{d i}\right)\right. \\
& \left.-\boldsymbol{\omega}_{i}^{\times} J_{i} \boldsymbol{\omega}_{i}+\boldsymbol{\Gamma}_{i}\right)
\end{aligned}
$$

Then, by substituting the control law given in (16) into (18) and after some manipulations, the next expression is obtained

$$
\dot{V}=s_{i}^{T}\left(-a_{i} \operatorname{sign}\left(s_{i}\right)-b_{i} s_{i}\right) \leq 0 \forall t \geq 0
$$

which assures the asymptotic stability of the system subjected to the proposed control law.

\section{B. Position Control Strategy for the VTOL Multi-Agent System}

The control strategy proposed inhere for a set of VTOLUAVs is intended to deal with the consensus problem. In other words, considering a virtual leader, the $i$ th follower must perform leader-following consensus as follows

$$
\lim _{t \rightarrow \infty}\left(p_{i}-p_{0}\right) \rightarrow 0
$$

where $p_{i}$ and $p_{0}$ are the position vectors of the $i$ th follower and the virtual leader, respectively.

Let the linear position dynamics of each aerial vehicle in the multi-agent system, expressed by (6), be rewritten as:

$$
\begin{aligned}
\left(\begin{array}{c}
\dot{p}_{x_{i}} \\
\dot{p}_{y_{i}} \\
\dot{p}_{z_{i}}
\end{array}\right)=\left(\begin{array}{c}
v_{x_{i}} \\
v_{y_{i}} \\
v_{z_{i}}
\end{array}\right), \\
\left(\begin{array}{c}
\dot{v}_{x_{i}} \\
\dot{v}_{y_{i}} \\
\dot{v}_{z_{i}}
\end{array}\right)=\left(\begin{array}{c}
\frac{U_{T_{i}}}{m_{i}}\left(\mathrm{C}_{\psi_{i}} \mathrm{~S}_{\theta_{i}} \mathrm{C}_{\phi_{i}}+\mathrm{S}_{\psi_{i}} \mathrm{~S}_{\theta_{i}}\right)+\varsigma_{x_{i}} \\
\frac{U_{T_{i}}}{m_{i}}\left(\mathrm{~S}_{\psi_{i}} \mathrm{~S}_{\theta_{i}} \mathrm{C}_{\phi_{i}}-\mathrm{C}_{\psi_{i}} \mathrm{~S}_{\phi_{i}}\right)+\varsigma_{y_{i}} \\
\frac{U_{T_{i}}}{m_{i}}\left(\mathrm{C}_{\phi_{i}} \mathrm{C}_{\theta_{i}}\right)-g+\varsigma_{z_{i}}
\end{array}\right)
\end{aligned}
$$

For control purposes, let the virtual control inputs be defined as follows

$$
\left\{\begin{array}{l}
V_{x_{i}}=\frac{U_{T_{i}}}{m_{i}}\left(\mathrm{C}_{\psi_{i}} \mathrm{~S}_{\theta_{i}} \mathrm{C}_{\phi_{i}}+\mathrm{S}_{\psi_{i}} \mathrm{~S}_{\theta_{i}}\right) \\
V_{y_{i}}=\frac{U_{T_{i}}}{m_{i}}\left(\mathrm{~S}_{\psi_{i}} \mathrm{~S}_{\theta_{i}} \mathrm{C}_{\phi_{i}}-\mathrm{C}_{\psi_{i}} \mathrm{~S}_{\phi_{i}}\right) \\
V_{z_{i}}=\frac{U_{T_{i}}}{m_{i}}\left(\mathrm{C}_{\phi_{i}} \mathrm{C}_{\theta_{i}}\right)-g
\end{array}\right.
$$

Hence, the desired Euler angles $\left(\theta_{d i}, \phi_{d i}\right)$ and the total thrust $U_{T_{i}}$ can be obtained as

$$
\left\{\begin{array}{l}
U_{T i}=m \sqrt{V_{x_{i}}^{2}+V_{y_{i}}^{2}+\left(V_{z_{i}}+g\right)^{2}} \\
\phi_{d i}=\arctan \left(\mathrm{C}_{\theta_{d i}}\left(\frac{V_{x_{i}} \mathrm{~S}_{\psi_{d i}}-V_{y_{i}} \mathrm{C}_{\psi_{d i}}}{V_{z_{i}}+g}\right)\right) \\
\theta_{d i}=\arctan \left(\frac{V_{x_{i}} \mathrm{C}_{\psi_{d i}}+V_{y_{i}} \mathrm{~S}_{\psi_{d i}}}{V_{z_{i}}+g}\right)
\end{array}\right.
$$

Thus, it follows that the representation of the system in (22) can be expressed as that of a disturbed system of the form:

$$
\left\{\begin{array}{l}
\dot{p}_{i}(t)=v_{i}(t) \\
\dot{v}_{i}(t)=u_{i}(t)+\varsigma_{i}(t)
\end{array}\right.
$$

where $u_{i}(t)$ is the control input and $\varsigma_{i}(t)$ corresponds to the external disturbance.
Now, let us define the lumped tracking errors for the $i$ th aerial vehicle as

$$
\begin{aligned}
& e_{p i}(t)=\sum_{j=1}^{N} a_{i j}\left(p_{s_{i}}(t)-p_{s_{j}}(t)\right)+b_{i}\left(p_{s_{i}}(t)-p_{0}(t)\right) \\
& e_{v i}(t)=\sum_{j=1}^{N} a_{i j}\left(v_{s_{i}}(t)-v_{s_{j}}(t)\right)+b_{i}\left(v_{s_{i}}(t)-\dot{p}_{0}(t)\right)
\end{aligned}
$$

The compact form of the lumped tracking error is given as

$$
\begin{aligned}
& e_{p}(t)=(\mathcal{L}+B) \otimes I_{3} \bar{p}(t) \\
& e_{v}(t)=(\mathcal{L}+B) \otimes I_{3} \bar{v}(t)
\end{aligned}
$$

where $e_{p}^{T}(t)=\left[e_{p 1}^{T}(t), \ldots, e_{p N}^{T}(t)\right]^{T}, \quad e_{v}^{T}(t)=$ $\left[e_{v 1}^{T}(t), \ldots, e_{v N}^{T}(t)\right]^{T}, \quad \bar{p}(t)=p(t)-1_{N \times 1} \otimes p_{0}(t)$, $\bar{v}(t)=v(t)-1_{N \times 1} \otimes \dot{p}_{0}(t), p(t)=\left[p_{1}^{T}(t), \ldots, p_{N}^{T}(t)\right]^{T}$, $v(t)=\left[v_{1}^{T}(t), \ldots, v_{N}^{T}(t)\right]^{T}, u(t)=\left[u_{1}^{T}(t), \ldots, u_{N}^{T}(t)\right]^{T}$, $\varsigma(t)=\left[\varsigma_{1}^{T}(t), \ldots, \varsigma_{N}^{T}(t)\right]^{T}$ and the term $\otimes$ denotes the Kronecker product.

Then, the time derivative of (27) can be further expressed by

$$
\begin{aligned}
& \dot{e}_{p}=e_{v} \\
& \dot{e}_{v}=H \otimes I_{3} \cdot\left(u(t)+\varsigma(t)-1_{N} \otimes \ddot{p}_{0}(t)\right)
\end{aligned}
$$

In order to meet the consensus control requirements for the VTOL-UAV's, a sliding surface is proposed as

$$
S_{i}(t)=e_{v i}(t)+\lambda_{i} e_{p i}(t)
$$

where $\lambda_{i}=\operatorname{diag}\left(\lambda_{i x}, \lambda_{i y}, \lambda_{i z}\right)$ is a matrix of control gains, and where $\lambda_{i}>0$. Let $S_{i}=\left[S_{1}^{T}, \ldots, S_{N}^{T}\right]^{T}$, then the compact form of (29) is given as

$$
S(t)=e_{v}(t)+\lambda e_{p}(t)
$$

According to [20], a sliding-mode control law consisting of $u_{i}(t)=u_{0 i}(t)+u_{w i}(t)$ where $u_{0 i}(t)$ takes care of the nominal part of the system and $u_{w i}(t)$ deals with the external disturbances such that $S_{i} \dot{S}_{i}<0$ can be designed. Let the control input be given by

$$
\begin{aligned}
u_{i}(t)=\left(l_{i i}+b_{i}\right)^{-1} & \left(\sum _ { j = 1 } ^ { N } \left(a_{i j} u_{j}(t)+b_{i} \ddot{p}_{0}(t)\right.\right. \\
& \left.\left.-\lambda_{i} e_{v i}(t)-\Pi_{i} \operatorname{sign}\left(S_{i}(t)\right)\right)\right)
\end{aligned}
$$

where $\Pi_{i}=\operatorname{diag}\left(\gamma_{i x}, \gamma_{i y}, \gamma_{i z}\right)$ is a matrix of adjustable control gains, and where $\gamma_{i}>0$.

Assuming that there exists a number $\Pi_{i}^{d}$, let $\varsigma_{\max }$ be the vector of lumped uncertainties, which is bounded as $\Pi_{i}^{d}>|\varsigma|$ with $\Pi_{i}^{d}=\operatorname{diag}\left(\gamma_{i x}^{d}, \gamma_{i y}^{d}, \gamma_{i z}^{d}\right)$ being the terminal solution for $\Pi_{i}$. To achieve $\Pi_{i}^{d}$, let the adaptive law be expressed as

$$
\dot{\Pi}_{i}=\varrho^{-1}\left|S_{i}(t)\right|
$$

with $\varrho=\operatorname{diag}\left(\rho_{i x}, \rho_{i y}, \rho_{i z}\right)$ a matrix of adaptive gains, defining also the adaptation speed and all subjected to $\rho_{i}>0$. Then, the compact form of (31) can be expressed by

$$
u(t)=\mathcal{H}^{-1} \otimes I_{3}\left(b \otimes \ddot{p}_{0}(t)-\lambda e_{v}(t)-\Pi \operatorname{sign}(S(t))\right)
$$

where $\Pi=\left[\gamma_{1}^{T}, \ldots, \gamma_{N}^{T}\right]^{T}$ and $S(t)=\left[S_{1}^{T}(t), \ldots, S_{N}^{T}(t)\right]^{T}$. 
The interest in the usage of event-driven systems is due to good performance in applications where resources are constrained. In multi-robot systems connected over a shared network, where rapid exchange of information is performed between agents, resources like bandwidth and processor times are constrained. Then, the event-based control is expected to offer better results. In this regard, the event-based control signals are updated only when a specific condition is satisfied, i.e. an event occurs. In consequence, traffic network is reduced or power consumption is minimised. With this in mind, the control law $u_{i}(t)$ given in (31) is modified in such a way that $\forall t \in\left[t^{k}, t^{k+1}\right)$

$$
\begin{aligned}
u_{i}(t)=\left(l_{i i}+b_{i}\right)^{-1} & \left(\sum _ { j = 1 } ^ { N } \left(a_{i j} u_{j}\left(t^{k}\right)+b_{i} \ddot{p}_{0}\left(t^{k}\right)\right.\right. \\
& \left.\left.-\lambda_{i} e_{v i}\left(t^{k}\right)-\Pi_{i} \operatorname{sign}\left(S_{i}\left(t^{k}\right)\right)\right)\right)
\end{aligned}
$$

Then, the errors introduced due to the discretization of the control are given by

$$
\begin{aligned}
& \bar{\epsilon}_{p}(t)=p\left(t^{k}\right)-p(t) \\
& \bar{\epsilon}_{v}(t)=v\left(t^{k}\right)-v(t)
\end{aligned}
$$

such that at $t^{k}, \bar{\epsilon}(t)=0$. Note that $t_{i}^{k}$ corresponds to the triggering instant of the $i$ th agent. Then, $\bar{\epsilon}_{v i}(t)$ and $\bar{\epsilon}_{v 0}(t)$ denotes the discretization error between the agents and leader, respectively. From (26),

$$
\begin{aligned}
& e_{p i}\left(t^{k}\right)=\sum_{j=1}^{N} a_{i j}\left(p_{s_{i}}\left(t^{k}\right)-p_{s_{j}}\left(t^{k}\right)\right)+b_{i}\left(p_{s_{i}}\left(t^{k}\right)-p_{0}\left(t^{k}\right)\right) \\
& e_{v i}\left(t^{k}\right)=\sum_{j=1}^{N} a_{i j}\left(v_{s_{i}}\left(t^{k}\right)-v_{s_{j}}\left(t^{k}\right)\right)+b_{i}\left(v_{s_{i}}\left(t^{k}\right)-\dot{p}_{0}\left(t^{k}\right)\right)
\end{aligned}
$$

Theorem 4.1: Considering the system described by (22) and (25), with error variables (26) and (35-37), sliding manifold $S(t)$ in the notions of sliding mode and the control law (34)

- The reachability of the sliding surface is confirmed for some reachability constant $\kappa>0$

- The event-based sliding mode control law (34) provides stability in the sense of Lyapunov if the adaptive gain $\Pi_{i}$ accomplishes

$$
\begin{aligned}
\Pi_{i}> & \sup \left\{\Upsilon+\bar{L}\left\|\bar{\epsilon}_{v i}(t)\right\|-\bar{L}\left\|\bar{\epsilon}_{v 0}(t)\right\|+\bar{L}\left\|\bar{e}_{v}\left(t^{k}\right)\right\|\right. \\
& \left.-\bar{L}\left\|e_{v}\left(t^{k}\right)\right\|\right\}
\end{aligned}
$$

where $\Upsilon=\varsigma_{\max }-\mathcal{H} \ddot{p}_{0}(t)+\ddot{p}_{0}\left(t^{k}\right)$

Proof: Let a candidate Lyapunov function be given by:

$$
V=\frac{1}{2} S(t)^{T} S(t)+\frac{1}{2} \tilde{\Pi}^{T} \varrho \tilde{\Pi}
$$

where the adaptation error is defined as $\tilde{\Pi}=\Pi-\Pi^{d}$. From, (39), the time derivative of $V$ is obtained as follows:

$\dot{V}=S^{T}(t)\left\{\mathcal{H} \otimes I_{3}\left(u(t)+\varsigma(t)-1_{N} \otimes \ddot{p}_{0}\right)+\lambda e_{v}(t)\right\}+\tilde{\Pi}^{T} \varrho \dot{\tilde{\Pi}}$
Then, by introducing the control law (34) in its compact form, the next expression is obtained

$$
\begin{aligned}
\dot{V}= & S^{T}(t)\left\{\mathcal { H } \otimes I _ { 3 } \left(\left(\mathcal { H } ^ { - 1 } \otimes I _ { 3 } \left(b \otimes \ddot{p}_{0}\left(t^{k}\right)-\lambda e_{v}\left(t^{k}\right)\right.\right.\right.\right. \\
& \left.\left.\left.\left.-\Pi \operatorname{sign}\left(S\left(t^{k}\right)\right)\right)\right)+\varsigma(t)-1_{N} \otimes \ddot{p}_{0}(t)+\lambda e_{v}(t)\right)\right\} \\
& +\tilde{\Pi}^{T} \varrho \dot{\tilde{\Pi}} \\
= & S^{T}(t)\left\{b \otimes \ddot{p}_{0}\left(t^{k}\right)-\lambda e_{v}\left(t^{k}\right)-\Pi \operatorname{sign}\left(S\left(t^{k}\right)\right)\right. \\
& \left.+\mathcal{H} \otimes I_{3}\left(\varsigma(t)-1_{N} \otimes \ddot{p}_{0}(t)+\lambda e_{v}(t)\right)\right\} \\
& +S^{T}(t)\left\{\Pi \operatorname{sign}\left(S\left(t^{k}\right)\right)-\Pi^{d} \operatorname{sign}\left(S\left(t^{k}\right)\right)\right\} \\
= & S^{T}(t)\left\{b \otimes \ddot{p}_{0}\left(t^{k}\right)-\lambda e_{v}\left(t^{k}\right)-\Pi^{d} \operatorname{sign}\left(S\left(t^{k}\right)\right)\right. \\
& \left.+\mathcal{H} \otimes I_{3}\left(\varsigma(t)-1_{N} \otimes \ddot{p}_{0}(t)+\lambda e_{v}(t)\right)\right\} \\
\leq & S^{T}(t)\left\{b \otimes \ddot{p}_{0}\left(t^{k}\right)-\lambda e_{v}\left(t^{k}\right)-\Pi^{d} \operatorname{sign}\left(S\left(t^{k}\right)\right)\right. \\
& \left.+\mathcal{H} \otimes I_{3}\left(\varsigma_{m a x}(t)-1_{N} \otimes \ddot{p}_{0}(t)+\lambda e_{v}(t)\right)\right\} \\
\leq & S^{T}(t)\left\{\Upsilon-\lambda e_{v}\left(t^{k}\right)-\Pi^{d} \operatorname{sign}\left(S\left(t^{k}\right)\right)+\mathcal{H} \otimes I_{3} \lambda e_{v}(t)\right\} \\
\leq & S^{T}(t)\left\{\Upsilon-\lambda e_{v}\left(t^{k}\right)-\Pi^{d} \operatorname{sign}\left(S\left(t^{k}\right)\right)+\mathcal{H} \otimes I_{3} e_{v}(t)\right\} \\
= & S^{T}(t)\left\{\Upsilon-\lambda e_{v}\left(t^{k}\right)-\Pi^{d} \operatorname{sign}\left(S\left(t^{k}\right)\right)\right. \\
& \left.+\mathcal{H} \otimes I_{3}\left(\bar{\epsilon}_{v i}(t)-\bar{\epsilon}_{v 0}(t)+\bar{e}_{v}\left(t^{k}\right)\right)\right\}
\end{aligned}
$$

Then, by applying the well-known Lipschitz continuity condition, the next expression can be obtained:

$$
\begin{aligned}
\leq & S^{T}(t)\left\{\Upsilon-\lambda e_{v}\left(t^{k}\right)-\Pi^{d} \operatorname{sign}\left(S\left(t^{k}\right)\right)\right. \\
& \left.+\mathcal{H} \otimes I_{3}\left(\bar{L} \bar{\epsilon}_{v i}(t)-\bar{L} \bar{\epsilon}_{v 0}(t)+\bar{L} \bar{e}_{v}\left(t^{k}\right)\right)\right\} \\
\leq & S^{T}(t)\left\{\Upsilon-\Pi^{d} \operatorname{sign}\left(S\left(t^{k}\right)\right)-\bar{L}\left\|e_{v}\left(t^{k}\right)\right\|\right. \\
& \left.+\mathcal{H} \otimes I_{3}\left(\bar{L}\left\|\bar{\epsilon}_{v i}(t)\right\|-\bar{L}\left\|\bar{\epsilon}_{v 0}(t)\right\|+\left\|\bar{L} \bar{e}_{v}\left(t^{k}\right)\right\|\right)\right\}
\end{aligned}
$$

As long as $S(t)>0$ or $S(t)<0$, then the condition $\operatorname{sign}(S(t))=\operatorname{sign}(S(t))$ is verified $\forall \in\left[t^{k}, t^{k+1}\right)$. Then, when the trajectories are outside the sliding manifold, (41) can be expressed as

$$
\begin{aligned}
\dot{V} \leq & \left\|S^{T}(t)\right\|\left\{\Upsilon-\Pi^{d}\left|S\left(t^{k}\right)\right|-\bar{L}\left\|e_{v}\left(t^{k}\right)\right\|\right. \\
& \left.+\left\|\mathcal{H} \otimes I_{3}\right\|\left(\bar{L}\left\|\bar{\epsilon}_{v i}(t)\right\|-\bar{L}\left\|\bar{\epsilon}_{v 0}(t)\right\|+\left\|\bar{L}_{v}\left(t^{k}\right)\right\|\right)\right\} \\
\Rightarrow \dot{V} \leq & -\kappa\left\|S^{T}(t)\right\|=-\kappa\|S(t)\|
\end{aligned}
$$

where $\kappa>0$ and $\Pi_{i}>\sup \left\{\Upsilon+\bar{L}\left\|\bar{\epsilon}_{v i}(t)\right\|-\bar{L}\left\|\bar{\epsilon}_{v 0}(t)\right\|+\right.$ $\left.\bar{L}\left\|\bar{e}_{v}\left(t^{k}\right)\right\|-\bar{L}\left\|e_{v}\left(t^{k}\right)\right\|\right\}$. It follows that the sliding manifold works as an attractor and the state trajectories converges towards it $\forall \in\left[t^{k}, t^{k+1}\right)$, which completes the proof of reachability.

The rest of the proof is not presented here, but it can be obtained following a similar procedure to that of the seminal work [17]. 
The time $t^{k}$ at which an event is triggered is described by a trigger mechanism. In other words, as long as a criterion (established by the trigger mechanism) is respected, the next event is not triggered and the control signal keeps its precedent constant value.

Corollary 4.2: Consider the group of mini aerial vehicles described by (21), with the control law (31). Let one assume the trigger mechanism is expressed as follows

$$
\xi=\left\|\nu_{1} e_{p i}+\nu_{2} e_{v i}\right\|-\left(r_{0}+r_{1} e^{-\varphi t}\right)
$$

with $\nu_{1}>0, \nu_{2}>0, r_{0} \geq 0, r_{1} \geq 0, r_{0}+r_{1}>0$ and $\varphi \in\left(0, \lambda_{2}(\mathcal{L})\right)$, where $\lambda_{2}(\mathcal{L})$ is the second eigenvalue if all the eigenvalues of $\mathcal{L}$ are arranged in ascending order.

Then, the trigger mechanism verifies the desired closed-loop behavior taking into account the error and its change rate [17].

Remark 4.3: The control law (31) allows the convergence to zero between the followers and the leader. However, if the consensus is extended to formation control with $\Lambda$ a feasible formation such that $\Lambda=\left[\mu_{i j} \in \mathbb{R} \mid \mu_{i j}>0 ; \quad i, j=1, \ldots, N\right]$ then, the tracking errors (26) can be rewritten as

$$
\begin{aligned}
e_{p i}(t) & =\sum_{j=1}^{N} a_{i j}\left(p_{s_{i}}(t)-p_{s_{j}}(t)-\mu_{i j}\right)+b_{i}\left(p_{s_{i}}(t)\right. \\
& \left.-p_{0}(t)-\mu_{i}\right) \\
e_{v i}(t) & =\sum_{j=1}^{N} a_{i j}\left(v_{s_{i}}(t)-v_{s_{j}}(t)\right)+b_{i}\left(v_{s_{i}}(t)-\dot{p}_{0}(t)\right)
\end{aligned}
$$

where $\mu_{i j}=\left\|\chi_{i}-\chi_{j}\right\|$ and $\mu_{i}=\left\|\chi_{i}-\chi_{0}\right\|$ describe the interagent and leader-follower distances and where $\chi_{1}, \ldots, \chi_{n} \in$ $\mathbb{R}^{3}$ are desired points.

An overview of the entire closed-loop system is depicted in Fig. 2

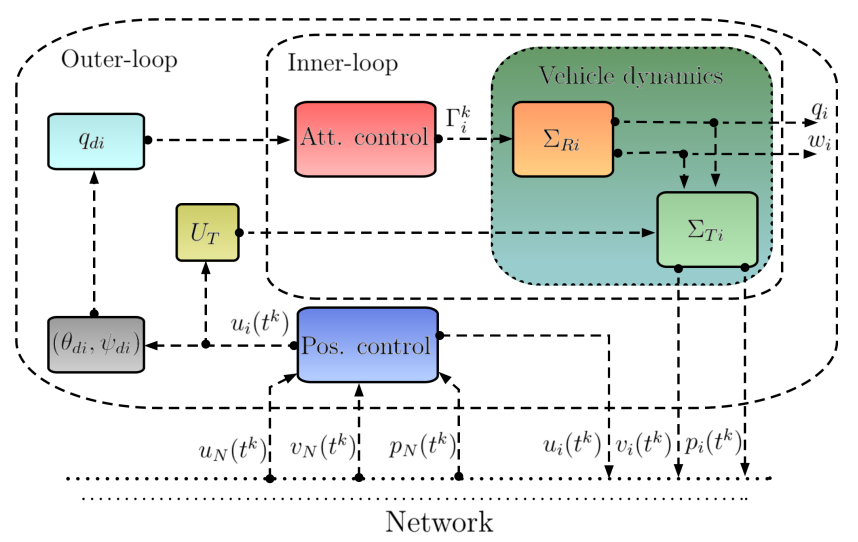

Fig. 2: Block diagram of the system.

\section{Simulation Results}

This section is devoted to the presentation of numerical simulation results to validate the proposed control strategy of a group of five VTOL aerial vehicles. The set of simulations was performed using the Matlab/Simulink ${ }^{\circledR}$ environment.

\section{A. Simulation Scenario}

The simulation model features the parameters depicted in Table I for each VTOL vehicle. Besides, for the case of study

\begin{tabular}{cccc} 
System & Description & Value & Units \\
\hline \multirow{5}{*}{ Quadcopter } & Mass (m) & 650 & $\mathrm{~g}$ \\
& Distance (d) & 17 & $\mathrm{~cm}$ \\
& Inertial moment $x\left(J_{\phi}\right)$ & 0.0075 & $\mathrm{Kg} \cdot \mathrm{m}^{2}$ \\
& Inertial moment $y\left(J_{\theta}\right)$ & 0.0075 & $\mathrm{Kg} \cdot \mathrm{m}^{2}$ \\
& Inertial moment $z\left(J_{\psi}\right)$ & 0.013 & $\mathrm{Kg} \cdot \mathrm{m}^{2}$ \\
\hline
\end{tabular}

TABLE I: Physical parameters for the VTOL vehicle

presented in this work, five aerial vehicles are considered $(N=5)$. The virtual leader $(N=0)$ shares to the neighbors its information related to the desired position or trajectory. The communication topology that is used for information exchange between the agents is shown in Fig. 3, where a directed configuration can be remarked. Besides, it can be seen that the information of the leader is acquired by all the agents in the system.

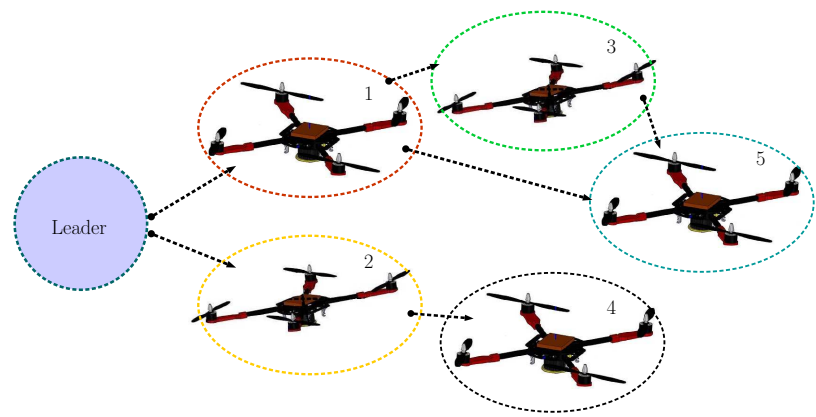

Fig. 3: Multi-VTOL system and communication flow.

The corresponding adjacency matrix $\mathcal{A}$ for the graph $G$, the incidence matrix $\mathcal{B}$ describing the connection of the leader with the neighbors and the matrix $\mathcal{L}+\mathcal{B}=\mathcal{H}$, corresponding to the closed-loop system, are given respectively as:

$$
\begin{gathered}
\mathcal{A}=\left[\begin{array}{lllll}
0 & 0 & 0 & 0 & 0 \\
0 & 0 & 0 & 0 & 0 \\
1 & 0 & 0 & 0 & 0 \\
0 & 1 & 0 & 0 & 0 \\
1 & 0 & 1 & 0 & 0
\end{array}\right], \mathcal{B}=\left[\begin{array}{lllll}
1 & 0 & 0 & 0 & 0 \\
0 & 1 & 0 & 0 & 0 \\
0 & 0 & 0 & 0 & 0 \\
0 & 0 & 0 & 0 & 0 \\
0 & 0 & 0 & 0 & 0
\end{array}\right] \\
\mathcal{H}=\left[\begin{array}{ccccc}
1 & 0 & 0 & 0 & 0 \\
0 & 1 & 0 & 0 & 0 \\
-1 & 0 & 1 & 0 & 0 \\
0 & -1 & 0 & 1 & 0 \\
-1 & 0 & -1 & 0 & 1
\end{array}\right]
\end{gathered}
$$

The eigenvalues of the matrix $\mathcal{H}$ are $1,1,1,1$ and 1 with multiplicity 5 . It is important to say that none of the eigenvalues is 0 , then the matrix $\mathcal{H}$ has full rank and there exists at least one spanning tree in the topology of Fig. 3.

The control and event function parameters used for the simulation can be found in Table II 

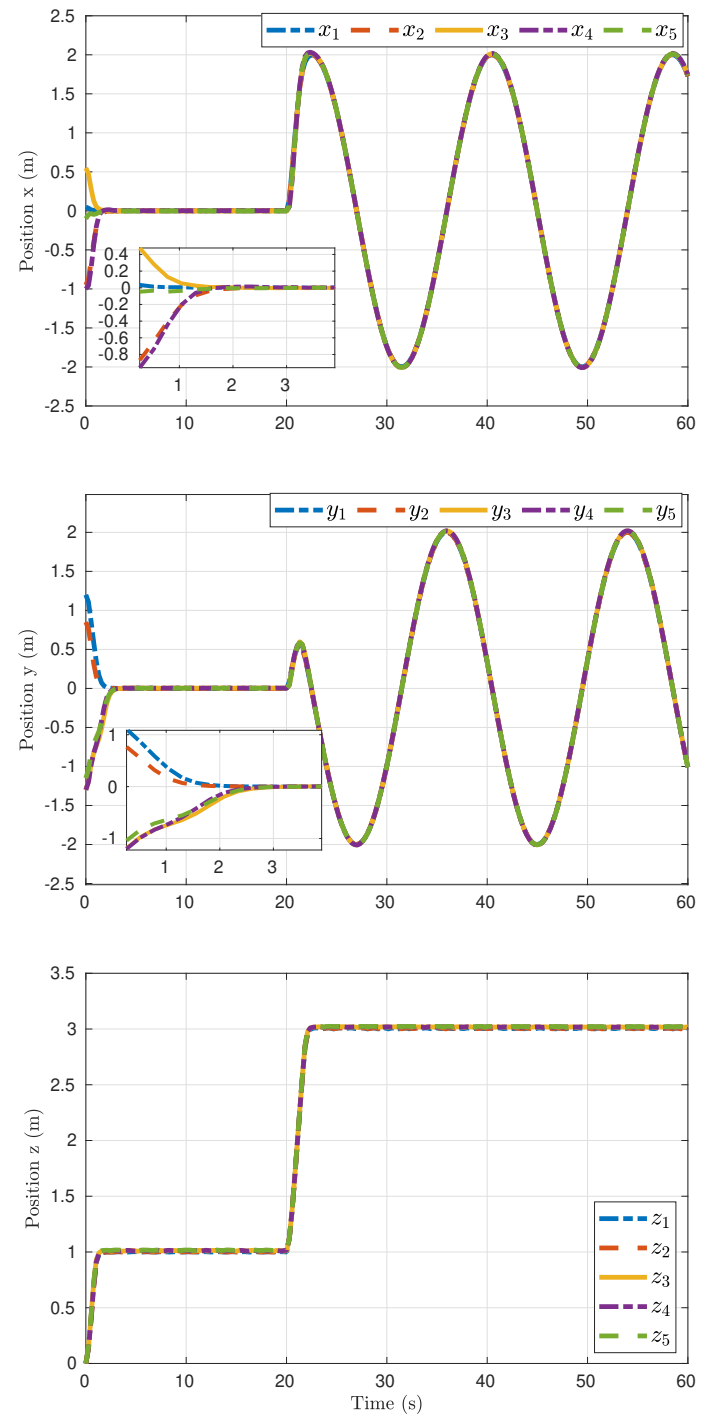

Fig. 4: Linear positions of the aerial vehicles during the consensus.

1) First Scenario: For the simulations, two scenarios were considered:

\begin{tabular}{ccc} 
Description & Parameter & Value \\
\hline Attitude controller & $\lambda_{\phi, \theta}$ & 2 \\
& $\lambda_{\psi}$ & 1.5 \\
& $K_{\phi, \theta, \psi}$ & 0.01 \\
& $a_{\phi, \theta}$ & 4.5 \\
& $a_{\psi}$ & 15 \\
& $b_{\phi, \theta, \psi}$ & 2 \\
Position controller & $\lambda_{i x, i y}$ & 3 \\
& $\lambda_{i z}$ & 4 \\
& $\rho_{i x, i y}$ & 2 \\
& $\rho_{i z}$ & 2.2 \\
Trigger mechanism & $\nu_{1 i}$ & 1 \\
& $\nu_{2 i}$ & 0.5 \\
& $r_{0 i}$ & 0.005 \\
& $r_{1 i}$ & 0.015 \\
& $\varphi$ & 0.2 \\
\hline
\end{tabular}

TABLE II: Numerical values for control laws and event function
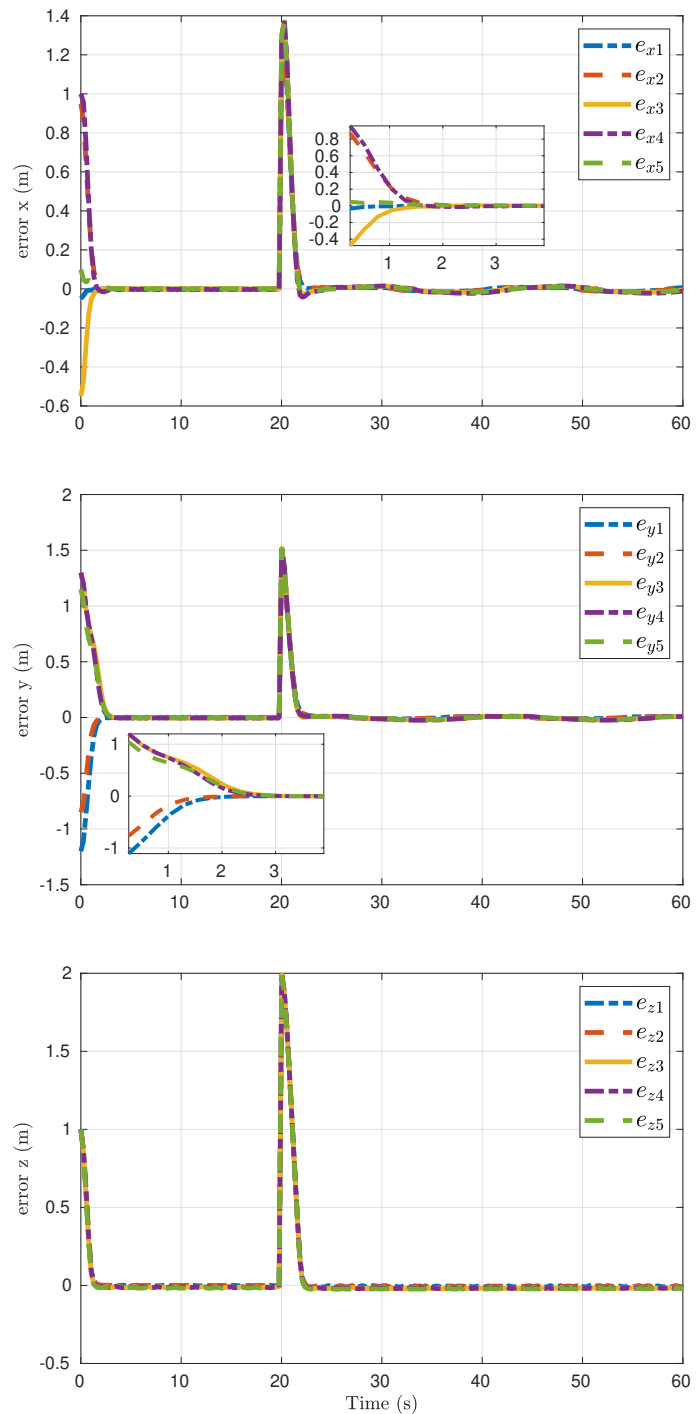

Fig. 5: Linear errors of the aerial vehicles during the consensus.

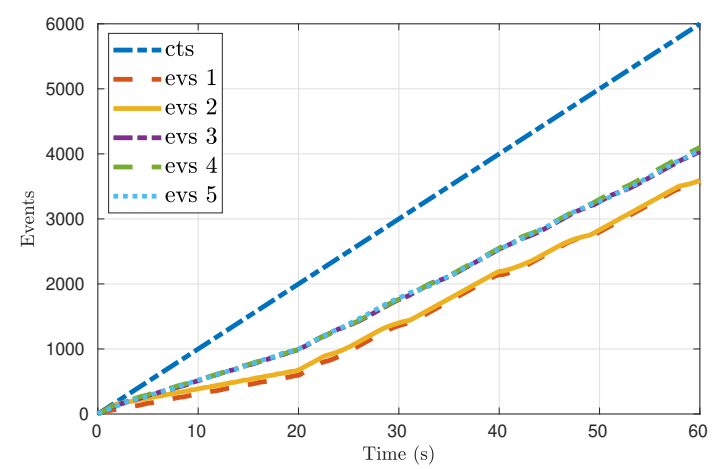

Fig. 6: Evolution of the events vs. continuous-time during the consensus.

- The behavior for the consensus of the group of multirotor aerial vehicles without the influence of a disturbance is studied. First, the multi-robot system is 

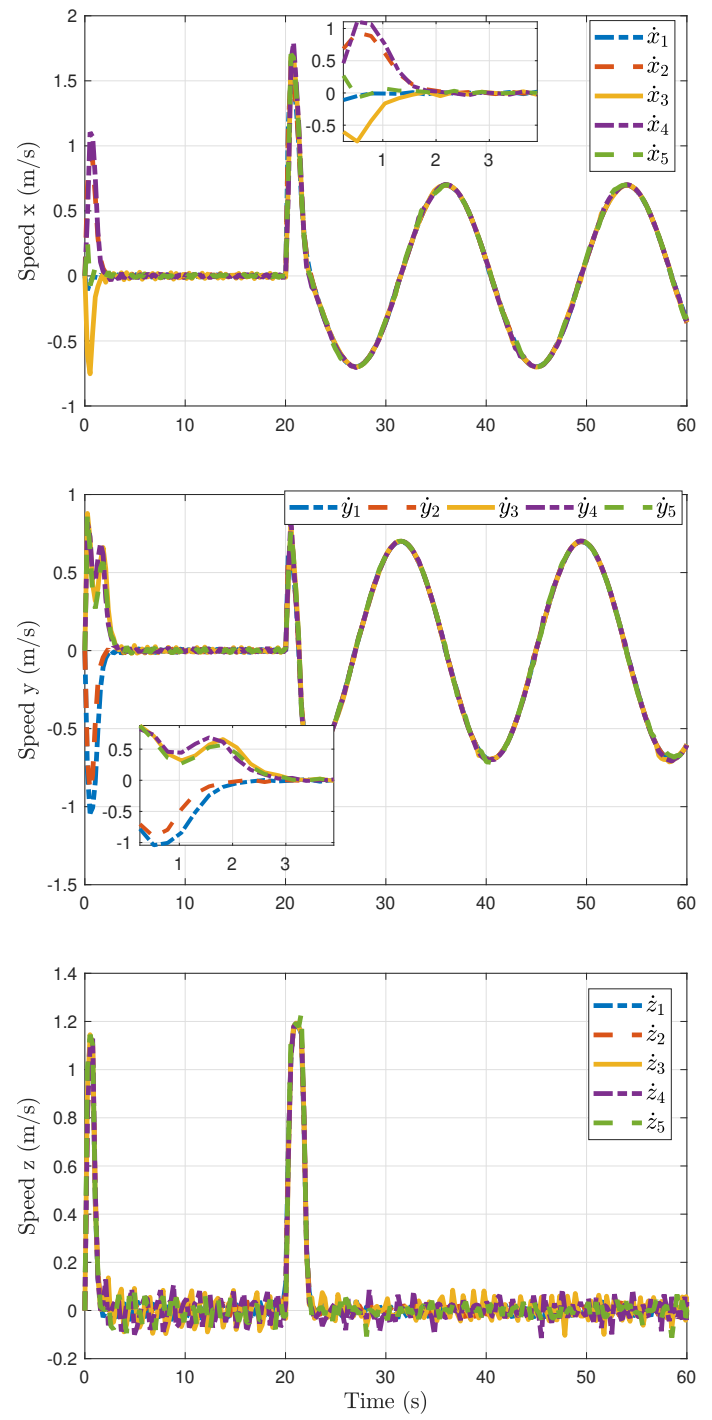

Fig. 7: Linear velocities of the aerial vehicles during the consensus.

initialized at orientation and $3 D$ positions given in the Table III. Then, the set of vehicles follows the virtual

\begin{tabular}{ccccc} 
VTOL MAS & $\psi_{i}$ & $p_{x_{i}}$ & $p_{y_{i}}$ & $p_{z_{i}}$ \\
\hline 1 & $1^{\circ}$ & $0.05 \mathrm{~m}$ & $1.25 \mathrm{~m}$ & $0.01 \mathrm{~m}$ \\
2 & $3^{\circ}$ & $-0.95 \mathrm{~m}$ & $0.85 \mathrm{~m}$ & $0.01 \mathrm{~m}$ \\
3 & $2^{\circ}$ & $0.55 \mathrm{~m}$ & $-1.3 \mathrm{~m}$ & $0.01 \mathrm{~m}$ \\
4 & $-1^{\circ}$ & $-1 \mathrm{~m}$ & $-1.22 \mathrm{~m}$ & $0.01 \mathrm{~m}$ \\
5 & $-1^{\circ}$ & $-0.1 \mathrm{~m}$ & $-1.15 \mathrm{~m}$ & $0.01 \mathrm{~m}$ \\
\hline
\end{tabular}

TABLE III: Initial conditions for the system

leader to the desired position given as $p_{0}=\left[\begin{array}{lll}0 & 0 & 1\end{array}\right]^{T} \mathrm{~m}$. After that, when the system is stabilized at time $t=20 \mathrm{~s}$, the virtual leader performs a trajectory described as $p_{0}=[2 \sin (2 \pi t / 16) 2 \cos (2 \pi t / 16) 3]^{T} \mathrm{~m}$.

- The behavior of the multi-agent system for the formation control under the influence of an unknown and timevarying disturbance is addressed. Indeed, the desired positions and trajectories given by the virtual leader,
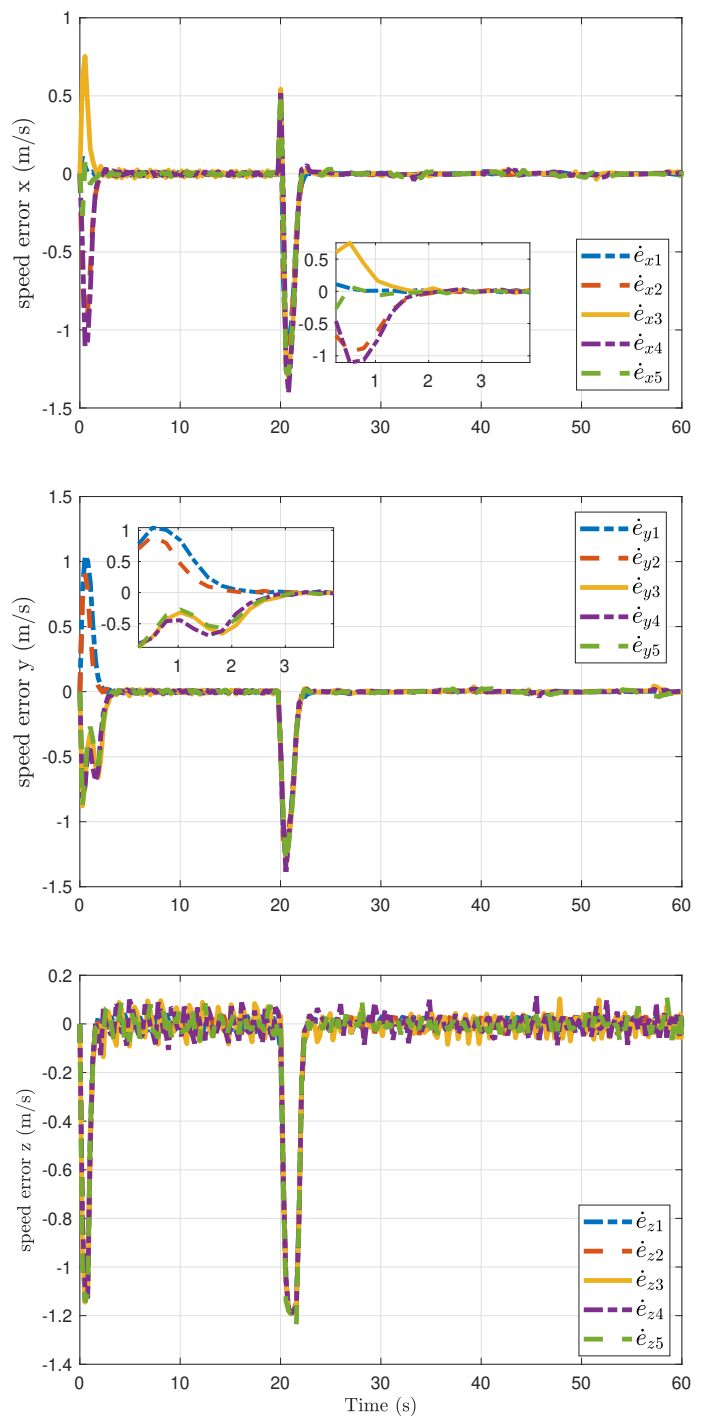

Fig. 8: Velocity errors of the aerial vehicles during the consensus.

as well as the initial conditions for the system, are the same as in the first scenario, however the multirobot system performs formation control, where the positions of the agents are intended to form a pentagon on the $x-y$ plane with a distance of $1.5 \mathrm{~m}$ between each vertex. The time varying disturbance is described by $\varsigma_{i}=$ $\left[\begin{array}{lll}0.4 \sin (0.1 \pi t) & 0.2 \cos (0.2 \pi t) & 0.1 \sin (0.15 \pi t)\end{array}\right]^{T} \mathrm{~N}$ and is present during the entire simulation. The simulation for both scenarios runs for $60 \mathrm{~s}$.

\section{B. Simulation Results}

Fig. 4 depicts the linear positions of the multi-agent system during the consensus. A numerical zoom was performed for the $x$ and $y$ axis during the first $4 \mathrm{~s}$ of the simulation, proving the consensus convergence in finite time. Fig. 5 shows the error profile of the follower agents. As in the first curves, a numerical zoom for the first seconds of simulation was performed to have a better perspective on how the error 
converges to zero quickly. This convergence to zero shows desirable closed-loop dynamics of the system and proves the effectiveness of the proposed control strategy. Fig. 7 and Fig. 8 show the linear velocities and the linear errors in terms of velocity of the different aerial vehicles during the simulation. The obtained results confirm the consensus convergence to the leader in terms of velocity. As before, numerical zooms were implemented to show more in detail the behavior of the multi-agent system. Finally, Fig. 6 shows how the events are triggered during the simulation. Using the triggering function given in (43), a minimal number of controller updates are expected and consequently the control effort is required only when necessary. The behavior of the events is clearly nonlinear, and from the results we can see that the number of updates increases during the trajectory-tracking phase.

1) Second Scenario: Fig. 9 depicts the behavior of the multi-VTOL system on the $3 d$ space for the formation control scenario. Fig. 10 shows the linear positions for each VTOL vehicle. An unknown and time-varying disturbance (previously described), acts over the system and as one can see, it corresponds to a matched disturbance, since the positions of the agents correspond to the expected ones, i.e. the trajectories are not affected. However, from Fig. 11 one can observe that the number of updates is slightly greater compared to the scenario when no disturbance is present. A comparison in the number of updates, when the disturbance is affecting the system or not, is presented in Table IV

\begin{tabular}{cccccc} 
VTOL agent & 1 & 2 & 3 & 4 & 5 \\
\hline Updates without disturbance & 3552 & 3591 & 4040 & 4099 & 4055 \\
Updates with disturbance & 3702 & 3637 & 4322 & 4284 & 4190 \\
\hline
\end{tabular}

TABLE IV: Control updates for scenarios 1 and 2 under the control law (34)

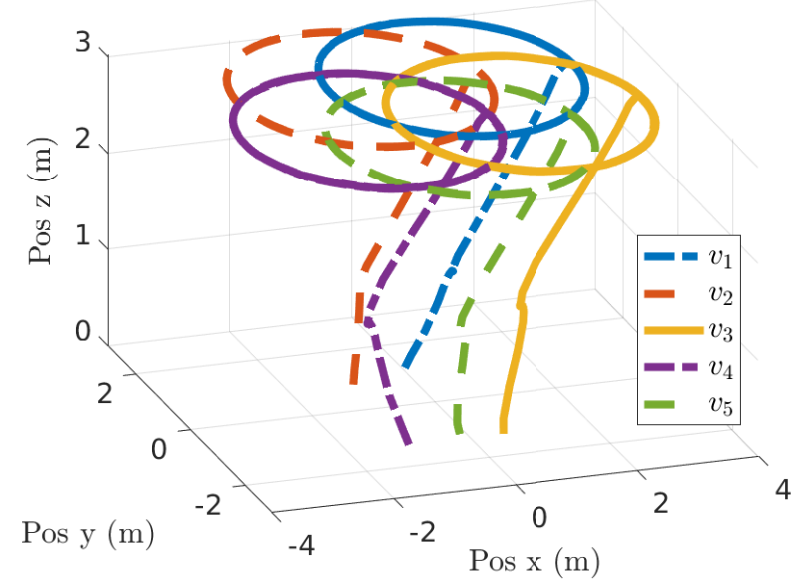

Fig. 9: Behavior of the multi-agent VTOL system in the $3 d$ space.

\section{CONCLUSIONS}

In this study, the consensus problem and formation control of a group of VTOL-UAVs has been addressed by means of a
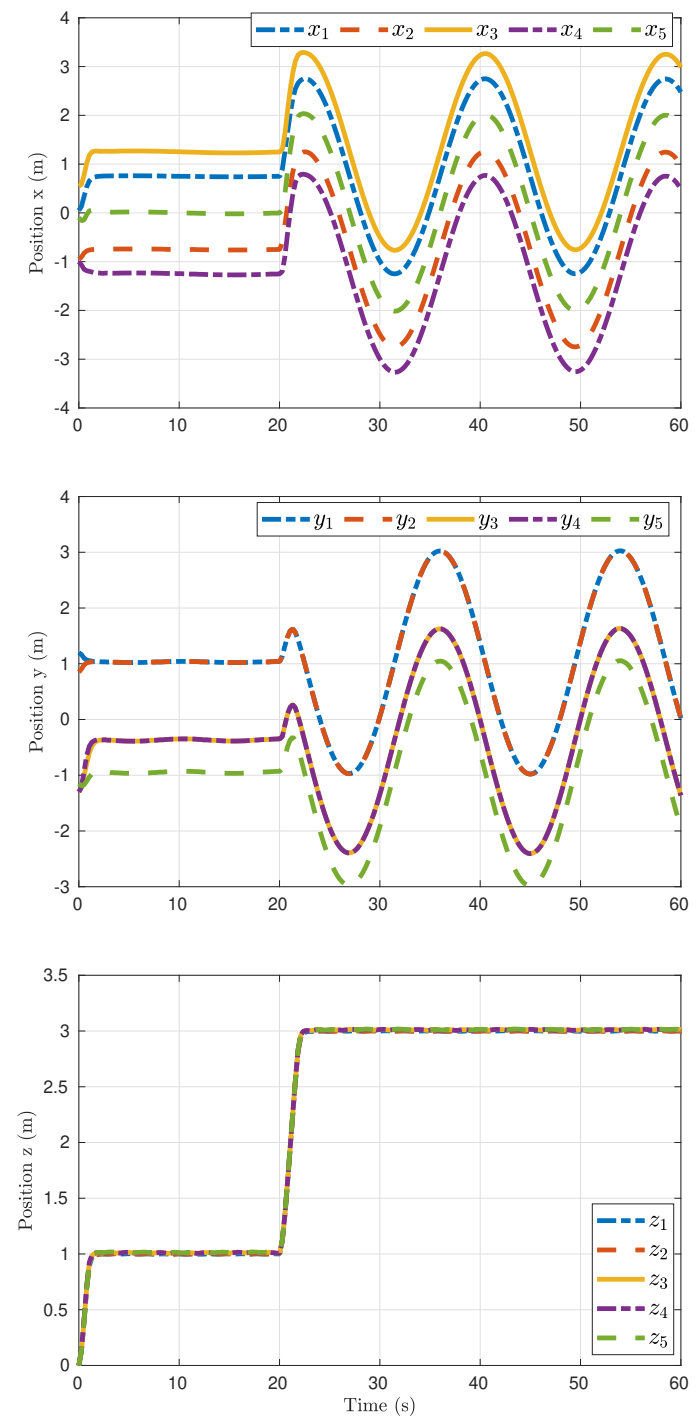

Fig. 10: Linear positions of the aerial vehicles during the formation.

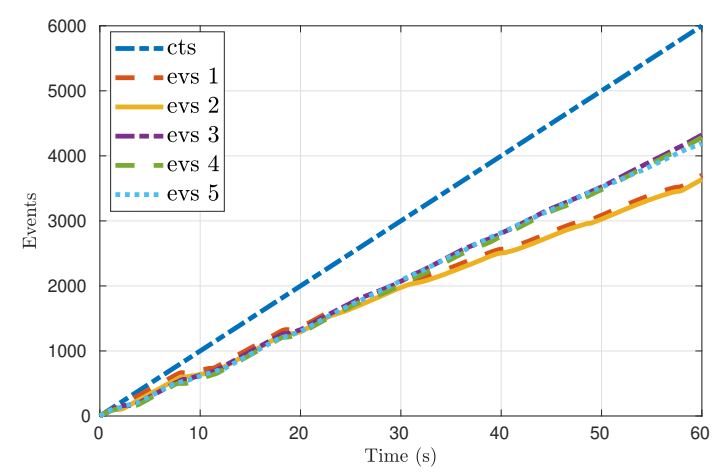

Fig. 11: Evolution of the events vs. continuous-time during the formation.

distributed and adaptive event-based sliding mode-control law. By integrating the robustness of the SMC with the benefits of the event-based scheme, closed-loop performance and low 
power computation were achieved. Due to the underactuated nature of the aerial vehicles, an inner-outer control loop methodology was implemented. The proposed attitude and multi-agent control laws were validated through stability analysis and numerical simulations. The simulations show that, even under the influence of unknown disturbances, the control law allows practical convergence to consensus or formation.

As future work, the design of an obstacle avoidance algorithm as well as the experimental implementation of the proposed strategies will be performed.

\section{REFERENCES}

[1] W. Huang, Y. Huang, and S. Chen, "Robust consensus control for a class of second-order multi-agent systems with uncertain topology and disturbances.", Neurocomputing, 2018, vol. 313, pp. 426-435.

[2] V. P. Tran, M. Garratt and I. R. Petersen, "Time-Varying Formation Control of a Collaborative Multi-Agent System Using NegativeImaginary Systems Theory", arXiv preprint arXiv:1811.06206, 2018.

[3] X. Dong, Y. Hua, Y. Zhou, Z. Ren, and Y. Zhong, "Theory and experiment on formation-containment control of multiple multirotor unmanned aerial vehicle systems," IEEE Transactions on Automation Science and Engineering, 2018, no 99, pp. 1-12.

[4] N. A. Lynch, "Distributed algorithms", Morgan Kaufmann, 1996.

[5] V. Borkar and P. Varaiya, "Asymptotic agreement in distributed estimation", in IEEE Transactions on Automatic Control, vol. 27, no. 3, pp. 650-655, June 1982.

[6] John Nikolas TSITSIKLIS, "Problems in decentralized decision making and computation", Massachusetts Inst of Tech Cambridge Lab For Information and Decision Systems, 1984.

[7] Y. Liu, J. M. Montenbruck, D. Zelazo, M. Odelga, S. Rajappa, H. H. Bülthoff, and A. Zell, "A distributed control approach to formation balancing and maneuvering of multiple multirotor UAVs", IEEE Transactions on Robotics, 2018, vol. 34, no 4, pp. 870-882.

[8] A. Abdessameud, "Formation Control of VTOL-UAVs Under Directed and Dynamically-Changing Topologies", 2019 American Control Conference, Philadelphia, PA, USA, 2019, pp. 2042-2047

[9] G. S. Seyboth, D. V. Dimarogonas and K. H. Johansson, "Event-based broadcasting for multi-agent average consensus", Automatica, 2013, vol. 49 , pp. $245-252$.
[10] C. Nowzari and J. Cortes, "Team-triggering Coordination for real-time control of networked cyber-physical systems", IEEE. Transactions on Automatic Control, 2016, vol. 61, pp. 34-47.

[11] Y. Dapeng, R. Wei, L. Xiangdond and Ch. Weisheng, "Decentralized event-triggered consensus for linear multi-agent systems under general directed graphs", Automatica, 2016, vol. 69, pp. 242-249.

[12] Z. Cai, H. Zhou, J. Zhao, K. Wu and Y. Wang, "Formation control of multiple unmanned aerial vehicles by event-triggered distributed model predictive control", IEEE Access, 2018, vol. 6.

[13] J. Guerrero-Castellanos, A. Vega-Alonzo, S. Durand, N. Marchand, V. R. Gonzalez-Diaz, J. Castañeda-Camacho and W. F. Guerrero-sanchez, "Leader-following consensus and formation control of VTOL-UAVs with event-triggered communications", Sensors, 2019, vol. 19, no. 24, pp. 5498.

[14] J. J. Castillo-Zamora, K. A. Camarillo-Gómez, G. I. Pérez-Soto and J. Rodríguez-Reséndiz, "Comparison of PD, PID and Sliding-Mode Position Controllers for V-Tail Quadcopter Stability", in IEEE Access, vol. 6, pp. 38086-38096, 2018.

[15] J. J. Castillo-Zamora, J. Escareno, I. Boussaada, O. Labbani and K. Camarillo, "Modeling and Control of an Aerial Multi-Cargo System: Robust Acquiring and Transport Operations", 2019 18th European Control Conference (ECC), Naples, Italy, 2019, pp. 1708-1713.

[16] W. Zhengyang, F. Qing and W. Bo, "Distributed Adaptive Sliding Mode Formation Control for Multiple Unmanned Aerial Vehicles", Chinese Control And Decision Conference (CCDC), 2020, pp. 2105-2110.

[17] R. K. Mishra and A. Sinha, "Event-triggered sliding mode based consensus tracking in second order heterogeneous nonlinear multiagent systems", European Journal of Control, 2019, vol. 45, pp. 30-44.

[18] Y. Deyin, L. Hongyi, L. Renquan and Sh. Yang, "Distributed SlidingMode Tracking Control of Second-Order Nonlinear Multiagent Systems: An Event-Triggered Approach", IEEE Transactions on Cybernetics, 2020, vol. 50, pp. 3892-3902.

[19] J. F. Guerrero-Castellanos, N. Marchand, A. Hably, S. Lesecq, and J. Delamare, "Bounded attitude control of rigid bodies: Real-time experimentation to a quadrotor mini-helicopter," Control Engineering Practice, 19(8), pp. 790-797.

[20] H. Ying-Jeh, K. Tzu-Chun and Ch. Shin-Hung, "Adaptive SlidingMode Control for Nonlinear Systems With Uncertain Parameters", IEEE Transactions on Systems, Man, and Cybernetics, 2008, vol. 38, pp. 534-539. 